\title{
Práticas alimentares dos lactentes
}

\author{
Gracimary Alves Teixeira1 ${ }^{1}$ Tainara Lôrena dos Santos Ferreira², \\ Jovanka Bittencourt Leite Carvalho ${ }^{3}$, Fábia Barbosa Andrade ${ }^{3}$
}

\section{RESUMO}

Objetivo: investigar as práticas de alimentação dos lactentes. Método: trata-se de uma pesquisa quantitativa e avaliativa, nas Unidades Básicas de Saúde, no período de maio a agosto de 2013, com 200 lactentes residentes no município de Santa Cruz-RN. Para coleta os sujeitos foram escolhidos mediante sorteio, utilizou-se questionário semi-estruturado, ambiente reservado e de forma individual. Na análise descritiva, utilizou-se dos programas SPSS para tabulação dos dados. Resultados: $34,5 \%$ deixaram 0 aleitamento materno antes dos dois anos, $79,5 \%$ fazem uso de água e $7 \%$ de chás, $22 \%$ comem salgadinhos, $25,5 \%$ bombons doces, $13 \%$ tomam refrigerantes. Conclusão: portanto, o aleitamento materno não está acontecendo em todas as crianças como preconiza o ministério da saúde, além disso, os alimentos açucarados e industrializados estão sendo introduzidos nos lactentes. Logo, medidas de educação em saúde precisam ser efetivadas pelos profissionais de saúde para obtenção da alimentação saudável desde as primeiras horas de vida.

Descritores: Aleitamento Materno; Nutrição do Lactente; Alimentação Complementar.

\section{Food practices of breastfed children}

\begin{abstract}
Objective: Investigating the practices of infant feeding. Method: It comes to a quantitative and evaluative research in Basic Health Units from May to August 2013, with 200 infants residing in Santa Cruz-RN city. To collect the subjects were chosen by lot and was used a semi-structured questionnaire in a booked individually environment. In the descriptive analysis, it was used the SPSS programs for data tabulation. Results: $34.5 \%$ stopped breastfeeding before two years; $79.5 \%$ used water and $7 \%$ teas; $22 \%$ ate snacks; $25.5 \%$, sweets candies; $13 \%$ took soft drinks. Conclusion: Therefore, breastfeeding is not happening to all children as recommended by the ministry of health, in addition, sugary and processed foods are being introduced in infants. Soon, measures of health education must be made by managers and health professionals with the mothers and family members to obtain healthy food from the first hours of life.
\end{abstract}

Descriptors: Breast Feeding; Infant Nutrition; Supplementary Feeding.

\footnotetext{
1 Especialista em Enfermagem Neonatal pela Faculdade Estácio do Rio Grande do Norte (FATERN), Natal, RN, Brasil.

${ }^{2}$ Acadêmica em Enfermagem na Universidade Federal do Rio Grande do Norte (UFRN), Caicó, RN Brasil.

${ }^{3}$ Doutora em Ciências da Saúde pela Universidade Federal do Rio Grande do Norte (UFRN), Caicó , RN, Brasil.
} 


\section{Introdução}

A amamentação exclusiva e a adequação nutricional dos alimentos complementares em tempo oportuno são fundamentais na prevenção da morbidade e mortalidade infantil, incluindo desnutrição e sobrepeso. Assim, atingir uma alimentação saudável para a maioria das crianças é uma estratégia essencial para assegurar a qualidade de vida de uma população".

O direito da mulher em amamentar seu filho está garantido pela Constituição Federal de 1998 (art.7, XVIII), o qual consiste em conceder, à mulher que deu à luz. Licença remunerada de 120 dias $^{2}$. E no Art. 396 da Lei n 5.452/43 trata que para amamentar o próprio filho, até este completar seis meses de idade, a mulher terá direito, durante a jornada de trabalho, a dois descansos especiais, de meia hora cada um³. E após a Lei no 11.770 de 9 de setembro de 2008 ser sancionada a licença passou a ser de 180 dias $^{4}$.

Esses direitos são medidas importantes de saúde pública com impacto efetivo na redução dos riscos a saúde dos indivíduos. Assim sendo, o ministério da saúde, preconiza o Aleitamento Materno Exclusivo (AME) até os seis meses de idade e a partir dos seis meses até os dois anos ou mais deve ser introduzida uma alimentação complementar para o infante 5 .

Nesse sentido, garantir o AME desde a primeira hora de vida extrauterina é a forma mais segura, eficaz e completa de alcançar crescimento e desenvolvimento saudável da criança. Além disso, estabelece um vínculo afetivo entre mãe e filho, criando uma ligação emocional que pode facilitar o desenvolvimento infantil ${ }^{6}$.

Os fatores nutricionais nas fases iniciais da vida podem ter repercussões, em curto e longo prazo, na saúde e no bem-estar do indivíduo, podendo determinar preferências alimentares posteriores, estendendo-se até a vida adulta, contribuindo para o aumento nos riscos de desenvolvimento futuro de doenças crônicas, tais como hipertensão arterial, diabetes, obesidade ${ }^{7}$.

Apesar de, a importância sobre o aleitamento materno ser um conhecimento científico de familiaridade dos profissionais de saúde, a amamentação persiste como importante preocupação da saúde pública, pois o desmame precoce, implica em maior risco de agravo à saúde da criança, aumentando os índices de morbidade e mortalidade infantil. Dessa forma emergiu a seguintepergunta norteadora do estudo: Qual a prática alimentar dos lactentes? Nesse contexto, o estudo tem o objetivo de investigar as práticas de alimentação dos lactentes.

\section{Metodologia}

Trata-se de uma pesquisa quantitativa e avaliativa realizada nas Unidades Básicas de Saúde que integram a rede da Atenção Primária à Saúde (APS), da cidade de Santa Cruz, Rio Grande do Norte, Brasil.

A coleta se deu no período de maio a agosto de 2013, com 200 mulheres, mães de crianças menores de dois anos, cadastradas nas 12 Equipes de Saúde da Família. Sendo as unidades de saúde e os sujeitos da pesquisa escolhidos mediante sorteio. Para tanto, foram respeitados os critérios de inclusão: ter mais de 18 anos; ser mães de criança com idade menor de dois anos; ter realizado pré-natal na unidade sorteada. Critérios de exclusão: não ser cadastrado na unidade de saúde; mães possuírem idade inferior a 18 anos, não possuir capacidade cognitiva para responder as perguntas.

A amostra compreendeu em 200 crianças menores de dois anos, residentes no município de Santa Cruz-RN, cadastradas nas 12 Equipes de Saúde da Família. Para coleta utilizou-se questionário semi-estruturado usado pelo Ministério da Saúde, e se deu em ambiente reservado e de forma individual, mediante explicação da pesquisa e assinatura do Termo de Consentimento Livre e Esclarecido pela mãe da criança. As entrevistas foram realizadas por alunos do curso de graduação de enfermagem UFRN/FACISA regularmente matriculados, previamente treinados. Em seguida foi realizada análise descritiva, utilizando-se dos programas SPSS para tabulação dos dados.

$O$ presente estudo trata-se de um recorte da pesquisa intitulada Morbidade e mortalidade materna e infantil: um estudo de avaliação na Atenção Primária à Saúde. Antes de iniciara a coleta foimantido contato junto a Secretaria Municipal de Saúde do referido município e com as Unidades de Saúde da Família, em seguida o projeto foi submetido ao Comitê de Ética em Pesquisa da Universidade Federal do Rio Grande do Norte para fins de apreciação e aprovação, conforme Resolução 466/2012 do Conselho Nacional de Saúde (CNS) sobre a pesquisa com seres humanos ${ }^{8}$. Com parecer favorável do comitê de ética: $n^{0} 311.613$. 


\section{Resultados e discussão}

A importância do aleitamento materno é bastante divulgada em estudos científicos, demonstrando suas propriedades nutricionais e inclusive imunológicas, atendendo as necessidades fisiológicas dos lactentes ${ }^{5,9}$. E o seu desmame precoce podem implicar em maior risco de agravos a saúde desta clientela ${ }^{10}$. Nesse estudo, $36,5 \%$ dos entrevistados são crianças de zero a seis meses e os outros $63,5 \%$ são lactentes de sete meses a menores de 2 anos. Conforme a tabela 1 , do total dessas crianças $65,5 \%$ faziam uso de leite materno em sua dieta, $34,5 \%$ deixaram o aleitamento materno antes dos 2 anos de idade e $55,5 \%$ faziam uso de outros tipos de leite que não são maternos. Segundo ROCCI; FERNANDES, 2014, estudo de coorte realizado em São Paulo com 225 puérperas apresentou que apenas $34,1 \%$ das mães aleitaram exclusivamente até os 180 dias ${ }^{11}$.

Contudo, o leite de vaca é considerado inapropriado para crianças menores de um ano, pois apresenta altas taxas de proteínas o que sobrecarrega os rins, tem baixa quantidade de carboidratos necessitando da adição de outros açúcares mais danoso a saúde, baixos níveis de vitaminas $C, D$ e $E$, dentre outras inadequações. $E$ na impossibilidade do leite materno deve-se utilizar formulas infantis que atendam as necessidades do lactente ${ }^{9}$.

Tabela 1 - Freqüência e Percentagem de aleitamento materno e consumo de outro tipos de leite em lactentes,

Santa Cruz, Estado do Rio Grande do Norte, Brasil, 2013

\begin{tabular}{c|c|c}
\hline Aleitamento & Frequência & $\%$ \\
\hline Leite materno & 131 & 65,5 \\
\hline Outros tipos de leite & 111 & 55,5 \\
\hline
\end{tabular}

No aleitamento materno exclusivo é desnecessário e inadequado a oferta de líquidos (chás, sucos e água) nos primeiros 6 meses de vida por reduzir o consumo de leite materno total e aumentar os riscos de morbimortalidade por diarréia ${ }^{12}$. Nesse estudo $36,5 \%$ lactentes tem de zero a 6 meses de idade e na tabela 2 observa-se que 79,5\% dos lactentes de zero a dois anos tomam água e $7 \%$ tomam chá, o que representa que algumas dessas crianças menores de 6 meses não tem seu aleitamento materno exclusivo. Corroborado por estudo transversal realizado em Campinas - SP, em que das 324 crianças 73,5\% foram inseridos líquidos precocemente ${ }^{13}$. Essa realidade revela a carência de atividades de educação em saúde e a qualidade da assistência de saúde, pois o oferecimento de líquidos aos lactentes com idade inferior aos 6 meses pode ser reduzida com apoio emocional, orientações precoces sobre o aleitamento materno durante o pré-natal e a visita puerperal14.

Tabela 2 - Freqüência e Percentagem de lactentes que fazem uso de água, chá, Santa Cruz, Estado do Rio Grande do Norte, Brasil, 2013.

\begin{tabular}{c|c|c}
\hline Oferta de líquidos & Frequência & $\%$ \\
\hline Água & 159 & 79,5 \\
\hline Chá & 14 & 7,0 \\
\hline
\end{tabular}

A OMS recomenda que a partir dos seis meses o leite materno deve ser mantido até os 2 anos ou mais, porém não deve ser exclusivo. Devem-se oferecer alimentos complementares e nutricionalmente adequados de forma gradual, inicialmente com as frutas em forma de papas doces, como lanches, depois as papas salgadas com verduras, cereais, carnes como almoço e jantar ambos servidos em colher; os sucos de frutas devem ser ofertados preferencialmente após as refeições principais e lembrar-se de ofertar água potável ${ }^{5,9}$.

Das crianças menores de dois anos, em estudo, $63,5 \%$ estão na faixa de alimentação complementar e as demais na faixa de aleitamento exclusivo. Ao investigar-se sobre sua alimentação observa-se, na tabela 3, que $58 \%$ tomam suco natural de frutas, $60 \%$ comem frutas, $49 \%$ verduras e legumes, $25 \%$ batata, mandioca ou inhame, $9 \%$ farinha de mandioca, $59 \%$ biscoito, pão, bolo, 53,5\% tomam mingau com leite não materno e 3,5\% tomam mingau sem nenhum tipo de leite. 
A partir do nono mês, respeitando-se a evolução da criança, deve-se passar gradativamente para a comida da família, pois nessa fase a maioria das crianças atinge seu estágio de desenvolvimento da mastigação, deglutição, digestão e excreção $0^{5,9}$. E o resultado apresentado ainda na tabela 3 foi que $61,5 \%$ das crianças em estudo se alimentam de comida de panela, um percentual inferior aos $63,5 \%$ que estão na faixa de alimentação complementar.

Tabela 3 - Freqüência e Percentagem de alimentação complementar em lactentes, Santa Cruz, Estado do Rio Grande do Norte, Brasil, 2013

\begin{tabular}{c|c|c}
\hline $\begin{array}{c}\text { Alimentos de } \\
\text { complementares }\end{array}$ & Freqüência & $\%$ \\
\hline Suco natural de frutas & 116 & 58,0 \\
\hline Frutas & 120 & 60,0 \\
\hline Legumes e verduras & 98 & 49,0 \\
\hline Mingau com leite & 107 & 53,5 \\
\hline Mingau sem leite & 7 & 3,5 \\
\hline Batata, mandioca, inhame & 50 & 25,0 \\
\hline Farinha de mandioca & 18 & 9,0 \\
\hline Biscoito, pão, bolo & 118 & 59,0 \\
\hline Comida de panela & 123 & 61,5 \\
\hline
\end{tabular}

É importante que os profissionais de saúde conheçam o conteúdo nutricional dos alimentos de ingestão das crianças e orientem os pais e/ou cuidadores para ofertar à criança alimentos ricos em vitaminas e evitar bebidas açucaradas, pois diminui o apetite por outros alimentos que seriam adequados a sua nutrição $0^{5,9}$.

Sabendo-se que o aleitamento materno pode proteger as crianças contra o sobrepeso e a obesidade ${ }^{15} \mathrm{e}$ que se deve evitar alimentos industrializados tais como refrigerantes, embutidos, enlatados ${ }^{5,9}$. Nesse contexto, a tabela 4 apresenta que $22 \%$ comem salgadinhos e $25,5 \%$ bombons doces, $13 \%$ tomam refrigerantes, pois são alimentos que fazem parte da dieta das famílias.

Tabela 4 - Frequência e consumo de alimentos industrializados em lactentes, Santa Cruz, Estado do Rio Grande do Norte, Brasil, 2013.

\begin{tabular}{c|c|c}
\hline $\begin{array}{c}\text { Alimentos } \\
\text { Industrializados }\end{array}$ & Freqüência & $\%$ \\
\hline Salgadinhos & 44 & 22,0 \\
\hline Bombons doces & 51 & 25,5 \\
\hline Refrigerantes & 26 & 13,0 \\
\hline
\end{tabular}

Sabemos que mudar radicalmente os hábitos de alimentação da família é uma tarefa bastante complexa, fazendo-se necessário a execução de um trabalho contínuo dos profissionais de saúde, com o intuito de orientar e evitar futuros transtornos no que diz respeito ao crescimento e desenvolvimento da criança.

No entanto, a educação em saúde implementadas as gestantes e às puérperas podem aumentar a autoeficácia materna em amamentar, podendo resultar em boas taxas de aleitamento materno ${ }^{16}$ e consequentemente reduzirem a mortalidade infantil, por causas evitáveis como diarréias e infecções respiratórias, além disso, diminuir os riscos de alergias, obesidade, hipertensão, colesterol alto e diabetes ${ }^{5}$. 


\section{Considerações Finais}

Os resultados deste estudo permitiram conhecer as características alimentares dos lactentes, nas quais se constatou o desmame precoce do aleitamento materno entre os lactentes e que este ainda não está acontecendo em todas as crianças de forma exclusiva até os 6 meses e complementada até os 2 anos, além disso os alimentos açucarados e industrializados estão sendo introduzidos desde essa idade. Práticas essas podem concorrer para o surgimento de doenças preveníveis na infância e desenvolvimento futuro de doenças crônicas.

Isso revela a necessidade de atividades de educação em saúde, que promovam a alimentação saudável, desde as consultas de prénatal até a puericultura, onde as propostas do Ministério da Saúde devem ser implementadas no âmbito da Atenção Primária à Saúde.

Assim, faz-se necessário a supervisão continuada nos processos de cuidado, bem como a busca ativa das famílias e crianças que necessitam retornar aos serviços de saúde, para receber orientações e um plano assistencial para cada realidade social e cultural. Isso concorre para a efetivação das diretrizes operacionais da Atenção Primária à Saúde e fortalecimento do Sistema Único de Saúde nos pilares de promoção da saúde e prevenção de doenças.

\section{Referências Bibliográficas}

1. Kaufmann, CC. Alimentação nos primeiros três meses de vida dos bebês de uma coorte na cidade de Pelotas, Rio Grande do Sul. Rev. paul. pediatr. 2012 jun; 30(2):157-165

2. Brasil. Presidência da República. Casa Civil. Constituição Federal de 1998. Ato das disposições constitucionais transitórias. [online] 1998. [acesso em 2014 agost 12]. Disponível em: http://www.planalto.gov.br/ccivil_03/constituicao/constituicaocompilado.htm

3. Brasil. Ministério do trabalho. Justiça do Trabalho. Direito do Trabalho. Lei $n^{0} 5.452$ de $1^{0}$ de maio de 1943. Aprova a consolidação das leis do trabalho. [online] 1943. [acesso em 2014 agost 12]. Disponível em:http://www.planalto.gov.br/ ccivil_03/decreto-lei/del5452.htm

4. Brasil. Lei n. 11.770, de 9 de setembro de 2008. Dispõe sobre a criação do Programa Empresa Cidadã, destinado à prorrogação da licença-maternidade mediante concessão de incentivo fiscal. Diário Oficial da Republica Federativa do Brasil. [online] 10 set. 2008. Seção 1.[acesso em 2014 agost 12]. Disponível em:http://www.planalto.gov.br/ccivil_03/_ ato2007-2010/2008/lei/l11770.htm

5. Brasil. Ministério da Saúde. Secretaria de Atenção à Saúde. Departamento de Atenção Básica. Saúde da criança: nutrição infantil: aleitamento materno e alimentação complementar. Brasília: 2009. p. 112.

6. Pontes AM, Lucena KDT, Silva ATMC, Almeida LR, Deininger LSCD. As repercussões do aleitamento materno exclusivo em crianças com baixo peso ao nascer. Saúde debate, 2013 Jun; 37(97):354-361.

7. Caetano MC, Ortiz TT, Silva SGL, Souza FIS, Sarni ROS. Alimentação complementar: praticas inadequadas em lactentes. ArchPediatrUrug 2012; 83(3): 226-232.

8. Ministério da Saúde (Brasil). Conselho Nacional de Saúde. Resolução 466 de 23 de dezembro de 2013. Aprova as diretrizes e normas regulamentadoras de pesquisa envolvendo seres humanos. Brasília (DF); 466. [citado em 07 jun 2013]. Disponível em: http://bvsms.saude.gov.br/bvs/saudelegis/cns/466/res466_23_12_2013.html.

9. Sociedade Brasileira de Pediatria. Departamento de Nutrologia. Manual de orientação: alimentação do lactente, alimentação do pré-escolar, alimentação do escolar, alimentação do adolescente, alimentação na escola. São Paulo: 2006. p. 64 .

10. Monteiro JCS, Gomes FA, Stefanello J, Nakano AMS. Leite produzido e saciedade da criança na percepção da nutriz durante o aleitamento materno exclusivo. Texto contexto - enferm. 2011 Jun, 20(2):359-367.

11. Rocci E; Fernandes RAQ, 2014. Dificuldades no aleitamento materno e influência no desmame precoce. Rev Bras Enferm. 2014 jan/fev; 67(1): 22-7.

12. Niquini, RP, Bittencourt SA, Lacerda EMA, Oliveira MIC, Leal MC. Acolhimento e características maternas associados à oferta de líquidos a lactentes.Rev. Saúde Pública [online]. 2010 Jun; 44(4):677-685.

13. Mais LA, Domene SMA, Barbosa MB, Taddei JAAC. Diagnóstico das práticas de alimentação complementar para 0 matriciamento das ações na Atenção Básica. Ciência\&SaúdeColetiva, 2014; 19(1):93-104

14. Sousa AM, Fracolli LA, Zoboli EL.Family practices related to breast-feeding maintenance: literature review and metasynthesis. Rev Panam Salud Publica. 2013 Aug; 34(2):127-134. 
15. Simon VGN,Souza JMP, Souza SB.Aleitamento materno, alimentação complementar, sobrepeso e obesidade em pré-escolares.Rev. Saúde Pública [online]. 2009, 43(1):60-69.

16. Dodt RCM, Ferreira AMV, Nascimento LA, Macêdo AC, Joventino ES, Ximenes LB.Influência de estratégia de educação em saúde mediada por álbum seriado sobre a autoeficácia materna para amamentar. Texto contexto - enferm. [online]. 2013, 22(3):610-618.

\section{Gracimary Alves Teixeira}

Endereço para correspondência - Av. Gov. Juvenal Lamartine, nº 978, Ap 402-B, Tirol, CEP: 59.022-020, Natal, RN, Brasil.

E-mail: gracimaryalves@yahoo.com.br

Lattes: http://lattes.cnpq.br/6486235205921142

Tainara Lôrena dos Santos Ferreira - tainara_lorena@hotmail.com Jovanka Bittencourt Leite Carvalho - jovanka@ufrner.br

Fábia Barbosa Andrade - fabiabarbosabr@yahoo.com.br

Enviado em 20 de agosto de 2014. Aceito em 29 de junho de 2015. 